\title{
UNIVERSIDAD ESTATAL A DISTANCIA: SIMIENTE FECUNDA QUE LA PATRIA POR TI COSECHÓ
}

\author{
Maricruz Corrales Mora ${ }^{1}$
}

\begin{abstract}
Resumen
El presente artículo es una remembranza alegórica de lo que significa el título de Institución Benemérita de la Educación y la Cultura Costarricense para la Universidad Estatal a Distancia, mediante un breve recorrido de momentos y datos que la autora ha querido resaltar de su historia. A diferencia de otras publicaciones en esta revista, la autora también se ha tomado la libertad de estructurar su alegoría, a partir de la metáfora de una cosecha; idea que para el ser costarricense, como pueblo con orígenes campesinos, provoca en esta Costa Rica del siglo XXI, alguna nostalgia del orgullo ancestral heredado.
\end{abstract}

\section{Palabras claves:}

$\bullet$ Educación a distancia $\bullet$ Benemeritazgo $\bullet$ Historia institucional $\bullet$ Educación $\bullet$ Cultura

\begin{abstract}
This article is one allegory to remember what means for UNED the national award given as one merit and acknowledged Institution in Costarrican Education and Culture. This written offers brief remembrances and data in order to focus on some details about UNED's history. The author uses the cropping allegory for structuring the article because she finds out on it one approach to rescue the rural roots and heritage related with the arisen and developing of UNED.
\end{abstract}

Key words:

$\bullet$ Distance education $\bullet$ State Merit Institution Award •Institutional history $\bullet$ Education $\bullet$ Culture

${ }^{1}$ Catedrática de la UNED. Investigadora, docente y autora en el área de Tecnología Educativa e Innovación. 


\section{SIMIENTE}

...Que la simiente dé el fruto, que el fruto dé la simiente, y que siempre, eternamente, el tiempo, pastor enjuto, pastoree dulcemente nuestra razón, nuestra mente

y se cobre su tributo.

(Simiente, Rafael Reyes López de Neira)

Entre las variedades posibles de educación existentes, la educación a distancia se ha transformado en los últimos lustros (sobre todo para la formación universitaria), en una que presenta enorme potencial para ser cultivada en diferentes espacios y circunstancias (UNESCO, Marzo 2006; Instituto del Banco Mundial, 2008).

En sus primeros años, la educación a distancia fue concebida para cubrir las formas distintas de estudio en las cuales no se da una presencia continua ni una supervisión inmediata de los profesores o maestros a sus estudiantes (como es usual hacerlo en las aulas tradicionales), sin embargo, los estudiantes se benefician de la planificación, guía y asesoría educativa de una organización que así lo hace (Holmberg, 1977; citado por Keegan, 1980). Por eso, también se le vio como una forma industrializada de enseñanza y aprendizaje, porque es un método de impartir conocimiento, habilidades y actitudes, el cual es racionalizado por la aplicación de una división del trabajo y principios organizacionales, así como por el uso extensivo y técnico de los medios, especialmente para el propósito de reproducir materiales de enseñanza de alta calidad que hacen posible enseñar a gran número de estudiantes al mismo tiempo, donde quiera que ellos vivan (Peters, 1973; ibídem).

Luego de una veintena de años de observación, investigación y reflexión sobre las prácticas de la enseñanza y el aprendizaje a distancia, el concepto de lo que realmente implica y en qué consiste este tipo de educación pudo mejorarse y es cuando se le concibe como un sistema de enseñanza y aprendizaje, cuya distancia física y temporal, entre quienes aprenden y enseñan, se resuelve mediante procesos interactivos de comunicación. Estos procesos son básicamente de cuatro tipos: estudiante - contenidos, estudiante - docente, estudiante - estudiante (éstas tres relaciones definidas inicialmente por Moore en 1989) y estudiante-tecnologías (definida posteriormente por Hillman, Willis y Gunawardena, en 1994).

La semilla, mejor conocida, proviene de la primera universidad que la asumió como su forma educativa: la Open University del Reino Unido (http://www.open.ac.uk); creada en la década de los sesentas del siglo pasado (http://www.open.ac.uk/about/ou/p3.shtml), pero cuyos orígenes se pueden remontar a principios de éste, al igual que otras experiencias similares a nivel mundial (la experiencia por correspondencia en China, Estados Unidos, Escandinavia o Australia). Desde entonces, conocida como la Open (o simplemente la OU), esta universidad se convirtió en una pauta, de primera categoría, para desarrollar similares experiencias en la propia Europa, en Asia, Oceanía y en América y, muchos de los académicos que se formaron en los primeros años de su proceso educativo, llegaron a ser consultores y asesores internacionales, para las nuevas adecuaciones y formas de implantarla a nivel mundial. 
Es a partir del éxito que cosechó la Open en su país, pero también de la aceptación del público para atender y reconocer una práctica educativa válida y efectiva (Moore y Kearsley, 1996), que la simiente de la educación a distancia se afianzó y empezó a dar buenos frutos, en la formación de nuevos profesionales, de distintas latitudes: en 1972 -en España- con la Universidad Nacional de Educación a Distancia; en 1974 -en Alemania- con la FernUniversität de Hagen y -en Pakistán- con la Allama Iqbal Open University; en 1979 -en China- con la creación del sistema nacional de Radio y TV Universidades (RTVUs); para compartir tan solo algunos ejemplos.

En Costa Rica, su cultivo se gestó a partir de 1975 (Ramírez, 2006), en la administración de gobierno del Lic. Daniel Oduber Quirós, a quien se le atribuye la paternidad del proyecto de ley que fundamentó el Plan Nacional de Desarrollo de la Educación Superior y la oportunidad de que el Estado tuviera un sistema informativo que utilizara los medios de comunicación para difundir sus fines, pero que también sirviera como sistema de apoyo para la educación y como medio para difundir la cultura.

\section{CULTIVO}

...Hay que sembrar un árbol, un ansia, un sueño, un hijo. Porque la vida es eso: ¡Sembrar, sembrar, sembrar! (Sembrar, José Ángel Buesa)

La historia ha venido a destacar la visión futurista del entonces presidente Oduber Quirós para la sociedad costarricense, ya que fue quien propició una serie de proyectos de los que estuvo en mucho pendiente, desde su formulación. Por ejemplo, su gobierno fue gestor del simposio "La Costa Rica del año 2000"; de la Ley del Consumidor; de la aceleración de las exportaciones no tradicionales a países fuera del área centroamericana; de la Ley de Desarrollo Social y Asignaciones Familiares; de la Ley de Incentivos Turísticos que dio base para el turismo ecológico; del cambio de La Sabana a un Parque Metropolitano Recreativo; de la creación del Sistema Nacional de Radio y Televisión y de la UNED, ambos para extender la educación a todo el territorio nacional (http://www.pln.or.cr/galeria/daniel.htm).

Por otra parte, la Costa Rica de los años setenta era una sociedad que venía cambiando de un modelo agrario de desarrollo, a uno industrial, a pasos lentos pero seguros: una disminución sustancial del trabajador del agro no calificado; una fuerte tendencia de aumentar los cuadros profesionales y técnicos, administrativos, comerciantes y vendedores, artesanales y similares; una cobertura amplia de la educación primaria gratuita que también empezaba a gozar de una importante conclusión de estudios a ese nivel (alrededor de 35\% en zonas urbanas); y una creciente concentración de los mejores servicios de desarrollo social en la región metropolitana ampliada del país (Rodríguez, Espinosa y Mora, 2002).

Asimismo, la población había aumentado notoriamente, ya que a partir del período 1962-1967 se presenta un incremento importante en los nacimientos, que supera los 60,000 anuales, que luego se reducirían a unos 53,000 en el año 1973 (INEC, 2006a). Costa Rica, para 1976, ya había excedido los dos millones de habitantes (INEC, 2006b). También, los datos de los censos nacionales indican que la población urbana, en las décadas de los $50 \mathrm{~s}$ y 60 s no superaba el 35\%, 
por lo que en realidad se tenía un país mayoritariamente rural. Es solo a partir de los 70 s que se empieza a revertir esta situación, con un claro desplazamiento a la zona urbana, que dará lugar en el 2000, a que se establezca en la gran área metropolitana el 57,5\% de la población (MIDEPLAN, Agosto 2002).

Igualmente, el país venía construyendo importantes bases y estructuras educativas: más escuelas y colegios, además de facilitar la formación de educadores, la emisión de una Ley Fundamental de la Educación (1957), la creación del Instituto Nacional de Aprendizaje - INA (1965), la creación del Instituto Tecnológico de Costa Rica (1971), la transformación de la Normal Superior (1968) en la Universidad Nacional (1973) y la creación de Consejo Nacional de Rectores - CONARE (1974). Y por otro lado, la población tenía acceso a la comunicación radial, a una incipiente industria televisa y a periódicos impresos.

Con estas condiciones nacionales y como España contaba con una institución que podía servir de modelo a la idea sugerida por el entonces Presidente de la República sobre el uso masivo de los medios, se encarga a don Enrique Obregón (funcionario universitario y del Ministerio de Educación Pública) visitar en 1974 la Universidad Nacional a Distancia de España, con la misión de firmar un convenio de cooperación, tendiente a conformar una primera red de televisión cultural y educativa. La elaboración del proyecto de ley se puso en las manos experimentadas del señor Fernando Volio, en ese momento Ministro de Educación Pública (Ramírez, 2006).

Pero habrían de pasar un par de años, para dar solución al reto planteado por la Presidencia. Se dieron más revisiones sobre las opciones e ideas iniciales, se realizaron nuevas consultas, audiencias y anteproyectos, además de otros viajes de verificación y asesoría, con el fin de consolidar un proyecto universitario a escala nacional, en el que se incluía la necesidad de contar con una universidad que pudiera atender la creciente demanda de educación superior, sin que por ello aumentaran considerablemente los costos asociados. Esto último estaría garantizado, a partir de las experiencias que se habían consolidado en educación superior a distancia en otros países, en las que se habían corroborado la promesa de alcanzar mayor eficiencia en el uso de los recursos de inversión educativa ${ }^{1}$.

Es así, como la educación a distancia vino a cambiar el horizonte educativo del país, a partir de su creación, en marzo de 1977 (La Gaceta, 12 de marzo de 1977). Ese día, la Universidad Estatal a Distancia (UNED) se comprometía a fortalecer los valores del Estado costarricense; en proporcionar educación superior mediante la utilización de técnicas de comunicación social, con métodos idóneos y flexibles a quienes no hubieren podido incorporarse al sistema formal universitario; a contribuir con la investigación científica para el progreso cultural, económico y social del país; a proporcionar instrumentos adecuados para el perfeccionamiento y formación permanente de todos los habitantes; a servir de vehículo para la difusión de la cultura y; a fomentar el espíritu científico, artístico, cultural y cívico del pueblo costarricense.

\section{FECUNDO TERRENO}

...Te daré cielo y tierra madurados.

Solo quiero semillas y semillas

(Diálogos de la siembra, Jorge Debravo) 
Sí. A don Daniel Oduber Quirós se le concede la paternidad de la Universidad Estatal a Distancia. Además, por los proyectos que ideó e hizo crecer, sus cualidades de gran orador y su visión de altura para el desarrollo del país, la UNED le rindió tributo adicional, con bautizar a su Paraninfo (el edificio de actos de la Universidad donde tienen lugar los acontecimientos importantes), con su nombre.

Sin embargo, también es necesario rescatar la visión y palabras de otros hombres, quienes ayudaron en su generación y propiciaron un suelo fértil para cultivar la educación a distancia. Por eso, es indispensable recordar la contribución de don Fernando Volio Jiménez, en la coordinación, defensa y formulación final del proyecto de ley de creación de la Universidad; del Lic. Enrique Obregón Valverde, quien hizo el contacto inicial y personal con el exrector de la UNED de España, para que luego se recibiera, de primera mano, la asesoría necesaria en la puesta en marcha del proyecto de ley y siguiera de cerca cada una de las fases del proyecto de ley; del diplomado Enrique Góngora Trejos y del Dr. Óscar Aguilar Bulgarelli, ambos miembros de la comisión convocada por el entonces Ministro Volio, quienes aportaron argumentos y datos para defender el proyecto de universidad a distancia y darle una organización inicial; del licenciado Eugenio Rodríguez Vega, quien fortaleció los fundamentos educativos que respaldarían el apoyo a la UNED como opción educativa de educación superior nueva y que fuera luego miembro externo del Consejo Universitario de la UNED y; del Dr. Francisco Antonio Pacheco Fernández, quien fue el responsable de iniciar las acciones de la UNED como Presidente de la Junta Universitaria y primer Rector.

Durante los primeros cinco años, la UNED fue organizada por una Junta Universitaria, cuyas funciones fueron afines a un Consejo Universitario y desde la cual, se dieron los primeros pasos, formas, estructuras, procesos y normas para la naciente universidad. La firma presidencial para el proyecto de ley se realizó el 3 de marzo de 1977 y la publicación en el diario oficial La Gaceta, el 12 de marzo siguiente.

La Junta Universitaria formuló, como parte de las funciones asignadas, un Estatuto Orgánico que sometió a una Asamblea Universitaria para su aprobación. Este sería el comienzo de la autonomía institucional formal que tendría la naciente universidad, pero no así de su autonomía financiera, la cual quedó inicialmente sujeta a la recaudación por la Ley del Timbre de Educación y Cultura, a las transferencias del Gobierno Central y a los ingresos propios; todos ellos bastante insuficientes. Es hasta 1978 que se aprobaría la adhesión de la UNED al Consejo Nacional de Rectores (CONARE), con lo cual pudo garantizarse, recibir financiamiento del Fondo Especial de Educación Superior (FEES), a partir de 1981.

La historia de germinación y el desarrollo consecuente de su quehacer en diferentes ramas y extensiones, como expresión del fecundo terreno que encontró en la sociedad costarricense, se describen a continuación.

Es 1978 y la UNED abre inicialmente la matrícula en 17 centros universitarios: San José, Puriscal, Quepos, Cartago, Alajuela, San Carlos, Zarcero, Palmares, Atenas, Nicoya, Cañas, Puntarenas, Ciudad Neilly, Palmar Norte, Pérez Zeledón, Limón y Siquirres. 
En el primer semestre lectivo registró 1284 estudiantes y en el segundo, 1020. Para abrir la matrícula, tiene que producir sus primeras cuatro unidades didácticas y más de 10 videos, de manera que los estudiantes pudieron contar con esos recursos, a diferencia de tener presentes a sus respectivos profesores.

En este primer encuentro con la sociedad costarricense, se hace notorio que la mayoría de la población es procedente de San José, pero también se puede apreciar un destacado número de Guanacaste, Puntarenas y Limón (entre 37\% y 34\%), así como más de 60\% de los estudiantes trabajan. Los programas de estudio fueron básicamente de Educación y de Administración (UNED, 1979, 1986).

Al siguiente año 1979, la UNED abre otros Centros Universitarios: Guápiles, Orotina, Río Frío y Arenal; crea una editorial educativa, su presupuesto llega alrededor de los 40 millones de colones y registra una planilla de 532 funcionarios, repartidos básicamente en tiempos completos (funcionarios de oficinas centrales) y de un cuarto de tiempo (tutores). En este año se empieza a notar que la población de estudiantes ligeramente ha aumentado y entre ellos, los solteros, además de quienes trabajan que llegan a más de 75\% (UNED, 1980).

En 1980, los datos recolectados en las matrículas sobre los estudiantes y sus condiciones revelan que la UNED ha podido consolidar 18 centros universitarios, a costa del cierre de algunos (Zarcero, Palmares, Atenas). También se destacan algunas regiones de procedencia de los estudiantes, que marcarán años después una consolidación de la Universidad: en San José (Cantón central, Desamparados, Goicoechea); en Alajuela (Cantón central y San Carlos); en Cartago (Cantón central); en Heredia (Cantón central); en Guanacaste (Liberia, Nicoya y La Cruz); en Puntarenas (Cantón central y Corredores) y en Limón (Cantón Central y Pococí). Para este año, se empieza a hacer evidente que un período lectivo es más numeroso que el otro; situación que se verá años después generalizado para los primeros períodos lectivos de cada año (UNED, 1981).

A los cinco años de apertura, en 1982, 54\% de la población estudiantil de la UNED es femenino, $61 \%$ con estado civil de solteros, 58\% trabajan mayoritariamente en el sector privado y un promedio de edad de 26 años, en matrículas que ascienden en los períodos académicos a 7,897 y 6,988; respectivamente. Es cuando también la UNED abre algunas librerías para extender el ingreso de recursos, pero igualmente, para difundir la educación y la cultura mediante su producción de libros de textos educativos y de literatura en general. Asimismo, empieza un esfuerzo institucional de fortalecer el acceso al conocimiento y a la lectura, mediante la organización de una biblioteca comunal, en cada uno de sus centros universitarios. Por vez primera, la UNED gradúa a 171 estudiantes y al año siguiente, se promoverá la Extensión Universitaria, con las primeras experiencias en Educación Ambiental (UNED, 1982, 1983).

En sus primeros diez años (1977-1987), hace ver que quienes no trabajan de su población estudiantil corresponden a mujeres, las cuales son amas de casa y que sus estudiantes empiezan a superar la formación que han tenido incompleta sus padres, equiparándose más bien a la que tiene el cónyuge. Más de $40 \%$ de los estudiantes trabajan en el sector privado y se mantiene que más de 55\% son solteros. Las provincias de San José y Alajuela son las más numerosas de estudiantes y fuera de ellas, destacan Liberia y Santa Cruz. Los Centros Universitarios han 
llegado a ser casi 30, clasificándolos en grandes (cuando tienen más de 500 estudiantes), medianos (cuando tienen entre 150 y menos de 499 estudiantes) y pequeños (los menores a 150 estudiantes). Se introduce el concepto de las guías didácticas de texto para coadyuvar en el proceso de tutoría y del aprendizaje de los estudiantes y, justo en sus diez años, alcanza a ser la segunda universidad nacional en número de estudiantes, cuando se le confía ser parte del Plan de Emergencia de formación de educadores (UNED, 1983, 1984, 1985, 1986, 1987, 1988).

De esta forma, la primera década revela una institución fortalecida con la confianza depositada de más de 10,000 estudiantes regulares (especialmente mujeres), que han visto en ella la opción de estudiar, sin tener que abandonar su trabajo, ni trasladarse a más de una hora en bus de su lugar de vivienda, ni dejar sus familias y en la cual pueden optar por formarse en diferentes énfasis de educación y administración, en administración agropecuaria o en administración de servicios de salud. También se revela que las mujeres son las que en mayor número y más pronto se gradúan.

En la siguiente década (1988-1997), la UNED vuelve a tener la tercera mayor población universitaria estatal (el convenio del Plan de Emergencia finaliza), llegando a más de 12,000 estudiantes y se aventura en sembrar la formación a distancia en áreas y zonas no cubiertas por el sistema educativo nacional. Por ejemplo, empieza con algunos de los centros penales, siendo el de La Reforma, el que más estudiantes atiende aún a la fecha; cierra y abre Centros Universitarios según la demanda estudiantil y las condiciones favorables para su instalación dentro de las comunidades que los acogen; abre su primera Maestría en Extensión Agrícola y se expande hasta la región de Talamanca para ofrecer algunos de sus programas.

Como opción para ampliar en general el acceso a la cultura, el programa de Estudios Universitarios inicia: una modalidad bastante abierta de formación a nivel universitario, para quienes se atrevan a definir prácticamente su propio programa de estudios.

Esta es una segunda década para demarcar diferencias con el resto de las otras universidades públicas, más allá de la principal, en su metodología educativa, pues tanto brinda una oportunidad mayor de ingreso a los estudiantes, que se da en cada uno de los períodos lectivos y no sólo al inicio de cada año, como también, en propiciar una Federación Estudiantil (a pesar de la posible incongruencia de la distancia como factor limitante para asociar a estudiantes).

En este período, la edad promedio de los estudiantes sube considerablemente, llega inclusive a los 32 años (en 1994), y por tanto, en esta década los estudiantes son mayoritariamente casados. Los datos hacen llamar la atención de que los profesionales del área agropecuaria ven en ella una opción de actualización y estudios. Continúa con una mayor predominancia de mujeres graduadas, provenientes de San José y Cartago y que entre todas suman un millar anual, como también de más hombres, quienes trabajan especialmente en el sector privado. Las evaluaciones de aprendizaje revelan que el rendimiento académico (un problema relacionado con la metodología a distancia), está asociado al grado de dificultad de la materia, al sistema de evaluación que emplee el curso, a la calidad de los materiales escritos y a la frecuencia y calidad de las tutorías que se ofrecen. Consolida su producción de recursos y materiales educativos, incorporando y elaborando programas televisivos y microprogramas reconocidos a nivel nacional como ATISBOS y recibiendo reconocimientos internacionales, como la donación de equipo 
audiovisual por parte del gobierno japonés. La UNED cierra la década con la primera organización de un congreso internacional en tecnología y educación a distancia, del que seguirán otros más programados en forma periódica, a modo de divulgar su quehacer universitario en la región centroamericana y para conocer los avances en la educación a distancia a nivel internacional (UNED, 1989, 1990, 1991, 1992, 1993, 1994, 1995, 1996, 1997, 1998).

En esta segunda década, la UNED empieza a contribuir a la formación de los cuadros profesionales del sector gubernamental, luego que en la primera década más bien lo hiciera prioritariamente con los del sector privado (acción no planificada, sino más bien dada por los estudiantes que la buscaron como casa de estudios) y se hace notorio que la Universidad se convierte en una opción educativa para propiciar el cambio de profesión u oficio, al confirmarse, en más de un año, la tendencia de que muchos de sus estudiantes no establecen relación alguna entre la ocupación y sus estudios. Esto en buena medida se venía dando con la incorporación de las amas de casa como estudiantes, pero igualmente se empieza a observar igual condición dentro de la población trabajadora. Sus graduados se establecen en más de un millar por período lectivo, básicamente a nivel de diplomados y bachilleratos universitarios. También, es la Universidad que se diferencia en buena parte de las demás estatales en que son sus propios estudiantes quienes se financian los estudios, teniendo como segunda opción al cónyuge y no los padres.

Al cumplir sus veinte años de creación, cabe destacar que la UNED atiende $21.8 \%$ de la población universitaria estatal de Costa Rica, con tan solo 7.2\% del FEES, demuestra históricamente el aprovechamiento de los recursos que se le han brindado y vuelve a ser la segunda universidad en matrícula estudiantil.

En la tercera década, la recién concluida (1998-2007), la UNED, junto a la población costarricense, ha variado. Primero, ha dejado la periodicidad semestral para pasar a cuatrimestres, y con ello, muchos de los estudiantes de primer ingreso han visto en ella la oportunidad de llevar, en menos de un año, la formación humanística de los Estudios Generales. Lo anterior, ha causado el efecto de reducir el promedio de años de la población estudiantil a 24 años. Se da una proporción casi similar de graduados en diplomado que en bachillerato, pero se aumentan considerablemente los niveles de licenciatura y de posgrado, inclusive a nivel de doctorado. Se introducen una serie de innovaciones tecnológicas como procesos de actualización de la educación a distancia, se destacan entre ellos, el uso intensivo de los recursos de la Internet, de videoconferencias y la modernización de la imprenta de la Editorial.

La Editorial de la UNED ha sido valorada como una empresa exitosa, tanto en sus volúmenes de producción como en la calidad de los contenidos de sus textos y en la selección de libros de línea editorial. De hecho, es un claro ejemplo de círculo virtuoso comercial, por cuanto dentro de la misma institución surgen las necesidades y demandas para la producción de libros $\mathrm{y}$ publicaciones, para que después se concreten y se distribuyen al público meta que los requiere.

También, durante este periodo, se brindan becas estudiantiles para facilitar el acceso a la educación universitaria. Los centros universitarios con mayor población estudiantil son los de San José, Cartago, Heredia, Alajuela y Palmares. Aumenta el número de los estudiantes que proviene de centros educativos nocturnos y colegios técnicos. La población prácticamente está 
dividida entre quienes estudian y trabajan (de nuevo más en el sector privado) o son amas de casa, y una vez más, se vuelve a dar una tendencia a que hayan más solteros. En esta década, los estudiantes cuentan con acceso a computadoras y a servicios de Internet, que no son propios, sino de sus trabajos o que alquilan en cafés-internet. El posgrado con mayor número de estudiantes y graduados es el de Administración de Servicios de Salud y luego la Maestría en Administración de Negocios. Se da la primera graduación de un doctorado y es mujer. Cuando se les pregunta a los estudiantes el porqué seleccionaron a la UNED para su formación, además de tener referencias positivas sobre ella de terceros o poderse costear mejor los estudios, también empiezan a aumentar las respuestas relacionadas con las obligaciones laborales. No obstante, también es necesario subrayar que, en los últimos años de esta tercera década, la población estudiantil ha llegado a ser mayoritariamente no laboral, más joven y con mayores competencias tecnológicas para atender sus estudios y demandarlos a la universidad y que, en todos estos años, el rendimiento académico de los grados no ha podido superar, en términos generales, el 65\% (UNED, 1999, 2000, 2001, 2002, 2003, 2004, 2005, 2006, 2007).

\section{COSECHA}

Ustedes los reconocerán por sus frutos. (San Mateo 7)

A marzo del 2008, la UNED ofrece 1 técnico, 3 profesorados, 10 diplomados, 29 bachilleratos y 18 licenciaturas en cuatro grandes áreas del conocimiento humano (Administración, Exactas y Naturales, Educación, Sociales y Humanidades); además 20 maestrías y 4 doctorados accesibles desde 33 centros universitarios distribuidos a lo largo y ancho del país. Tiene una matrícula promedio en sus cuatrimestres de más de 22,000 estudiantes, mantiene el segundo lugar en población estudiantil de la educación superior estatal y ha graduado en sus treinta años de labor, a más de 30,000 estudiantes. En realidad, una enorme cosecha de frutos educativos, de muy variada distribución y resultados.

Otro fruto relevante de esta cosecha ha sido la diversidad de producciones académicas, de diferente formato y medios que ha puesto la UNED a disposición de los estudiantes y la sociedad costarricense, para facilitar los procesos de enseñanza y aprendizaje a distancia, además de la consolidación de su producción de materiales educativos y del resto de publicaciones que tiene en su haber la Editorial UNED, por los cuales le han conferido más de una distinción y reconocimiento por parte de colegios profesionales, jurados de ferias de libros, asociaciones de industrias gráficas, premios nacionales de literatura en las ramas de novelas, cuentos, ensayos y otras obras, que sin lugar a dudas son referencias del aporte hecho a la cultura nacional.

Sin embargo, y con la modestia con la que ha trabajado la UNED durante treinta y un años, la cosecha más galardonada ha sido la que recibió en noviembre del 2007, mediante el decreto de ley No. 8623 de la Asamblea Legislativa (Diario La Gaceta No. 225, 22 de noviembre de 2007), cuando fue honrada a la altura del más alto título patrio de Costa Rica: ser Institución Benemérita de la Educación y la Cultura Costarricense.

Esta honrosa designación solo se asigna a aquellas organizaciones e instituciones que, en un período clave de su trayectoria, demuestran amplias cualidades, funciones y acciones que 
benefician y abarcan a una gran mayoría de ciudadanos. A la UNED la enaltecieron con este benemeritazgo, con tan solo treinta años de existencia, pero todo un largo recorrido de esfuerzo, cobertura y entrega de sus frutos a la educación y a la cultura nacional.

En el acto de firma de la Ley, la Presidencia de la República extendió su reconocimiento y homenaje a la UNED, en palabras del Dr. Oscar Arias, al declarar que "no sólo es la cuna de grandes actores de nuestra historia, sino que ha sido ella también actora indispensable del acontecer nacional durante los últimos 30 años. El reconocimiento que le hemos extendido, es por eso más que justo" (Arias, 11 de diciembre del 2007).

Sí. Actora cultural, democratizadora de la educación (especialmente universitaria) y propulsora del cambio social en las últimas tres décadas; aspectos claves que el diputado B. Venegas, expuso en el proyecto de ley para la declaración del benemeritazgo en los siguientes términos (Venegas, 8 de marzo del 2007):

...Ha brindado enormes oportunidades a personas que por condiciones socioeconómicas se han visto imposibilitadas para continuar con sus estudios cambiando de manera definitiva sus vidas. Sin duda alguna, la Universidad Estatal a Distancia incrementó las posibilidades de democratización y la universalización de la educación en Costa Rica, continuando su labor con la creación de órganos como el CONED (Colegio Nacional Estatal a Distancia). Durante estos años, la Universidad Estatal a Distancia ha formado a veintiocho mil setecientos cincuenta y cinco profesionales, incorporándolos al mercado laboral en todo lo amplio del territorio nacional. Cuenta, además, con treinta y cuatro sedes universitarias y dos subsedes, las cuales son de gran acceso para todos los habitantes de la República. Así también, brinda a la comunidad estudiantil materiales educativos de muy buena calidad y adaptados a las necesidades nuevas del entorno.

También, medios de comunicación nacionales rindieron tributo a la UNED, al señalar:

Justo y oportuno reconocimiento de la Asamblea Legislativa a la Universidad Estatal a Distancia (UNED). La UNED realiza, gracias a la democratización del conocimiento con calidad, el compromiso de la integración social. (La Nación, 24 de noviembre de 2007)

La UNED cuenta con 34 sedes en diferentes comunidades del país con el fin de favorecer el acceso a aquellas personas que por diversos motivos no pueden asistir a los centros de educación superior tradicionales y, de esa forma, se constituyan en agentes de desarrollo de sus propios pueblos. Esta universidad además brinda atención a estudiantes en todos los centros penales del país desde 1979 y en sus 30 años de existencia ha graduado 32 mil profesionales y, actualmente, cuenta con 24.650 estudiantes matriculados. (Al Día, 11 de diciembre de 2007)

UNED es institución benemérita... Ha graduado a 32 mil estudiantes en 30 años de labor. (Diario Extra, 12 de diciembre de 2007) 
Por todo eso, por la simiente de la educación a distancia sembrada hace más de treinta años en Costa Rica y la región, por la democratización de la educación superior, por las contribuciones que ha hecho en la difusión del conocimiento y el progreso cultural, económico y social del país y por ser un invaluable vehículo para la difusión de la cultura, que se le pueda seguir cantando, como su himno lo expresa, “.... simiente fecunda que la Patria por ti cosechó”.

Ahora, el reto es aún más grande que hace 30 años, con una visión renovada de la educación. Ahora, la UNED tiene, ante sí, las distancias no solo físicas, sino culturales y virtuales de nuestros tiempos y espacios de marginación y encuentro digitales. Ahora, es una vez más, el momento para repensarla, siempre desde la vocación humanista que la ha distinguido de muchos centros de enseñanza. Ahora, de nuevo es el tiempo para volver a actuar y brindar las mejores opciones con que se pueda acceder al aprendizaje y al conocimiento. Es tiempo de volver a la siembra.

\section{NOTA}

${ }^{1}$ Como no es interés de la autora distraerse, fuera de su alegoría, en los detalles de la gestación del proyecto, se invita a los lectores a conocer la primera parte sobre la Creación de la UNED, del libro escrito por el ex-rector, Dr. Celedonio Ramírez, La Tercera Revolución Educativa Costarricense. Memoria de la creación y puesta en marcha de la UNED (1975-1982), en donde están recopiladas interesantes y curiosas anécdotas de esos años, y se puede saber quiénes, cuántos y de qué forma contribuyeron a labrar la educación a distancia en el país (op. cit.).

\section{REFERENCIAS}

Al Día (2007, 11 de diciembre). UNED declarada institución benemérita. En Última Hora. San José, Costa Rica. Recuperado el 18 de marzo de 2008, de http://www.aldia.co.cr/ad ee/2007/diciembre/11/ultimahora1349038.html

Arias, O. (2007, 11 de diciembre). Discurso Presidencial. En Acto de homenaje a la UNED: Institución Benemérita de la Educación y Cultura Costarricense. San José, Costa Rica: Casa Presidencial de la República de Costa Rica.

Comisión con Potestad Legislativa Plena Tercera (2007, 19 de setiembre). Primer Debate. Expediente No. 16565. Declaración de la Universidad Estatal a Distancia como institución benemérita de la educación y la cultura de Costa Rica. En Acta de la Sesión No. 10. Segunda Legislatura. Segundo periodo de sesiones ordinarias. Asamblea Legislativa de la Republica de Costa Rica. San José, Costa Rica. Recuperado el 18 de marzo de 2008, de http://www.asamblea.go.cr/actas/comision/plena3/acta10.doc

Diario Extra (2007, 12 de diciembre). UNED es institución benemérita. En Nacionales. San José, Costa Rica. Recuperado el 18 de marzo de 2008, de http://www.diarioextra.com/2007/diciembre/12/nacionales10.php

Hillman, D.; Willis, B. y Gunawardena, C. (1994). Learner-interface interaction in distance education: an extension of contemporary models and strategies for practitioners. En American Journal of Distance Education, 8(2), 30-42. 
Instituto del Banco Mundial (2008). Red global de aprendizaje para el desarrollo (GDLN). En Aprendizaje. Recuperado el 19 de marzo de 2008, de http://www.bancomundial.org/aprendizaje/gdln.html .

Instituto Nacional de Estadísticas y Censos (2006a). Cuadro población. Series Tasa bruta de natalidad 1950-2006. Recuperado el 1 de marzo de 2008 de http://www.inec.go.cr/

--------. (2006b). Cuadro población de Costa Rica. San José, Costa Rica. Recuperado el 1 de marzo de 2008, de http://www.inec.go.cr/

Keegan, D. (1980). On defining distance education. En Distance Education, 1(1), 13-36. Recuperado el 19 de marzo de 2008 , de http://www.informaworld.com/smpp/content $\sim$ content $=\mathrm{a} 739147906 \sim \mathrm{db}=\mathrm{all}$

La Gaceta (1977, 12 de marzo). Ley de creación de la Universidad Estatal a Distancia. Decreto de la Asamblea Legislativa No. 6044. San José, Costa Rica: Imprenta Nacional.

. (2007, 22 de noviembre). Universidad Estatal a Distancia, declarada Benemérita de la Educación y Cultura de Costa Rica. Decreto de la Asamblea Legislativa No. 8623. San José, Costa Rica: Imprenta Nacional.

La Nación (2007, 24 de noviembre). Honores a la UNED. En Editorial/Opinión. San José, Costa Rica. Recuperado el 18 de marzo del 2008, de http://www.nacion.com/ln_ee/2007/noviembre/24/opinion1326972.html

Ministerio de Planificación y Política Económica (2002, agosto). Población total de Costa Rica por zona (urbano y rural) de residencia. Sistema de Indicadores sobre Desarrollo Sostenible (SIDES). Recuperado el 1 de marzo del 2008, de http://www.mideplan.go.cr/sides/ambiental/23-1.htm

Moore, M. (1989). Editorial: Three types of interaction. En The American Journal of Distance Education, $3(2), 1-7$.

Moore, M. G.; Kearsley, G. (1996). Distance education. A systems view. California, EE.UU.: Wadsworth Publishing Co.

Partido Liberación Nacional. Daniel Oduber Quirós. En Galería de Expresidentes. Recuperado el 19 de marzo de 2008, de http://www.pln.or.cr/galeria/daniel.htm

Ramírez, C. (2006). La tercera revolución educativa costarricense. Memoria de la creación y puesta en marcha de la UNED (1975-1982). San José, Costa Rica: EUNED.

Rodríguez, F.; Espinosa, R. y Mora, F. (2002). Evolución de la estructura social y conducta electoral en Costa Rica 1973-2000. [Documento PDF]. San José, Costa Rica: Publicaciones Series Censal INEC Costa Rica. Recuperado el 19 de marzo de 2008, de http://www.inec.go.cr/

Universidad Estatal a Distancia (1979). Anuario estadístico 1978. San José, Costa Rica: Centro de Investigación y Estadística.

. (1980). Anuario estadístico 1979. San José, Costa Rica: Centro de Investigación y Estadística. (1981). Anuario estadístico 1980. San José, Costa Rica: Centro de Investigación y Estadística. 
--------. (1982). Anuario estadístico 1981. San José, Costa Rica: Centro de Investigación y Estadística.

--------. (1983). Anuario estadístico 1982. San José, Costa Rica: Centro de Investigación y Estadística.

--------. (1984). Anuario estadístico 1983. San José, Costa Rica: Centro de Investigación y Estadística.

---------. (1985). Anuario estadístico 1984. San José, Costa Rica: Centro de Investigación y Estadística.

--------. (1986). Anuario estadístico 1985. San José, Costa Rica: Centro de Investigación y Estadística.

--------. (1987). Anuario estadístico 1986. San José, Costa Rica: Centro de Investigación y Estadística.

--------. (1988). Anuario estadístico 1987. San José, Costa Rica: Centro de Investigación y Estadística.

--------. (1989). Anuario estadístico 1988. San José, Costa Rica: Centro de Investigación y Estadística.

---------. (1990). Anuario estadístico 1989. San José, Costa Rica: Centro de Investigación para el Desarrollo de la Educación a Distancia.

--------. (1991). Estadísticas 1990. San José, Costa Rica: Centro de Investigación para el Desarrollo de la Educación a Distancia.

---------. (1992). Estadísticas 1991. San José, Costa Rica: Centro de Investigación para el Desarrollo de la Educación a Distancia.

- (1993). Estadísticas 1992. San José, Costa Rica: Centro de Investigación para el Desarrollo de la Educación a Distancia.

--. (1994). Estadísticas 1993. San José, Costa Rica: Centro de Investigación para el Desarrollo de la Educación a Distancia.

--. (1995). Estadísticas 1994. San José, Costa Rica: Centro de Investigación para el Desarrollo de la Educación a Distancia.

--------. (1996). Estadísticas 1995. San José, Costa Rica: Centro de Investigación para el Desarrollo de la Educación a Distancia.

---------. (1997). Estadísticas 1996. San José, Costa Rica: Vicerrectoría de Planificación.

---------. (1998). Estadísticas 1997. San José, Costa Rica: Vicerrectoría de Planificación.

--------. (1999). Estadísticas 1998. San José, Costa Rica: Centro de Investigación y Evaluación Institucional.

---------. (2000). Estadísticas 1999. San José, Costa Rica: Centro de Investigación y Evaluación Institucional.

--------. (2001). Estadísticas 2000. San José, Costa Rica: Centro de Investigación y Evaluación Institucional. 
---------. (2002). Estadísticas 2001. San José, Costa Rica: Centro de Investigación y Evaluación Institucional.

---------. (2003). Estadísticas 2002. San José, Costa Rica: Centro de Investigación y Evaluación Institucional.

---------. (2004). Estadísticas 2003. San José, Costa Rica: Centro de Investigación y Evaluación Institucional.

---------. (2005). Estadísticas 2004. San José, Costa Rica: Centro de Investigación y Evaluación Institucional.

UNESCO (2006). Open and distance learning forum. En A. Cameron (Comp.), International Internet Forum on the UNESCO Higher Education Open and Distance Learning Knowledge Base. Final Report. [Documento PDF]. Recuperado el 19 de marzo de 2008, de http://unesdoc.unesco.org/images/0014/001456/145634E.pdf

Venegas, B. (2007, 8 de marzo). Proyecto de Ley. Expediente No. 16565. San José, Costa Rica: Asamblea Legislativa de la República de Costa Rica. Recuperado de www.asamblea.go.cr/actas/comision/plena3/acta10.doc Benemeritazgo Asamblea Legislativa lista el 4 de diciembre del 2007. 